\title{
Inverse relationship between bioavailable testosterone and subclinical coronary artery calcification in non-obese Korean men
}

\author{
Byoung-Jin Park ${ }^{1}$, Jae-Yong Shim ${ }^{1}$, Yong-Jae Lee ${ }^{1}$, Jung-Hyun Lee ${ }^{2}$ and Hye-Ree Lee ${ }^{1}$
}

Although low testosterone levels in men have been associated with high risk for cardiovascular disease, little is known about the association between male sex hormones and subclinical coronary disease in men with apparently low cardiometabolic risk. This study was performed to investigate the association between male sex hormones and subclinical coronary artery calcification measured as coronary calcium score in non-obese Korean men. We examined the relationship of total testosterone, sex hormone-binding globulin, bioavailable testosterone and free testosterone with coronary calcium score in 291 non-obese Korean men (mean age: $52.8 \pm 9.3$ years) not having a history of cardiovascular disease. Using multiple linear regression, we evaluated associations between log (sex hormone) levels and log (coronary calcium score) after adjusting for confounding variables in 105 men with some degree of coronary calcification defined as coronary calcium score $\geqslant 1$. In multiple linear regression analysis, bioavailable testosterone was inversely associated with coronary calcium score $(P=0.046)$ after adjusting for age, body mass index, smoking status, alcohol consumption, regular exercise, mean blood pressure, resting heart rate, C-reactive protein, fasting plasma glucose, total cholesterol, triglyceride, high-density lipoprotein (HDL) cholesterol, hypertension medication and hyperlipidemia medication, whereas total testosterone, sex hormone-binding globulin and free testosterone were not $(P=0.674, P=0.121$ and $P=0.102$, respectively). Our findings indicate that bioavailable testosterone is inversely associated with the degree of subclinical coronary artery calcification in non-obese men. Asian Journal of Andrology (2012) 14, 612-615; doi:10.1038/aja.2012.19; published online 23 April 2012

Keywords: bioavailable testosterone; cardiovascular disease; coronary calcium score; male sex hormone; subclinical coronary artery disease; testosterone

\section{INTRODUCTION}

Cardiovascular disease (CVD) is one of the leading causes of death around the world, especially in developed countries. ${ }^{1}$ The impending aging of societies augments the importance of CVD as a factor that both decreases the quality of life and increases the burden on society. ${ }^{1}$ Thus, identification of risk factors for CVD and its early detection may be important in providing a means to prevent or slow its progression. ${ }^{2}$

A non-invasive marker of early coronary arterial wall alteration is now available. The coronary calcium score (CCS) measured by multidetector computed tomography (MDCT) is a sensitive marker of coronary artery atherosclerosis and reflects the severity of coronary artery stenosis to some extent. ${ }^{2,3}$ Recent epidemiological evidence suggests that CCS could help identify patients with cardiovascular risks in addition to the Framingham risk score. ${ }^{4}$

An increasing body of evidence suggests that low testosterone levels in men are associated with high cardiovascular risk. ${ }^{5-7}$ Although many studies have been focused on the association between testosterone and CVD events as well as mortality, and the free testosterone level has been reported to be inversely associated with degree of coronary artery disease in men with clinical coronary artery disease, ${ }^{8}$ little is known about the association between sex hormones and subclinical coronary disease in men with apparently low cardiometabolic risk.

Therefore, we examined the associations of male sex hormones with subclinical coronary artery calcification as measured by CCS in nonobese Korean men.

\section{MATERIALS AND METHODS}

Study sample

We reviewed the medical records of 328 males who voluntarily participated in a physical examination including male sex hormones and MDCT at the Health Promotion Center of Gangnam Severance Hospital, Yonsei University College of Medicine in Seoul, Korea, between October 2007 and November 2008.

The inclusion criteria were community-dwelling men with body mass index (BMI) below $25 \mathrm{~kg} \mathrm{~m}^{-2}$, which is a cutoff value for generalized obesity in Asia. ${ }^{9}$ Subjects meeting any of the following criteria were excluded $(n=37)$ : a history of testosterone therapy and/or a history of diabetes, angina pectoris, myocardial infarction or cerebrovascular diseases. After the exclusion, 291 participants were included 
in the final analysis. This study was approved by the Institutional Review Board of Yonsei University College of Medicine in Korea.

The examinations were performed by medical staff according to standard procedures. The participants were asked about lifestyle behavior (including exercise, cigarette smoking and alcohol consumption) and about ongoing treatment for any disease. If the participants were under treatment, they were asked for the date of diagnosis and a list of current medications. Trained staff reviewed the completed questionnaires and entered the responses into a database. The participants were classified as non-smokers, ex-smokers or current smokers and were categorized by alcohol consumption as non-drinkers or abstainers (alcohol drinking $<140$ g per week) and current drinkers (alcohol drinking $\geqslant 140$ g per week). Regular exercise was defined as more than twice a week. Body weight and height were measured to the nearest $0.1 \mathrm{~kg}$ and $0.1 \mathrm{~cm}$, respectively, with subjects wearing light indoor clothing and no shoes. BMI was calculated as the ratio of weight to height squared $\mathrm{kg} \mathrm{m}^{-2}$. Blood pressure and resting heart rate were measured with the participants in the sitting position after 5 minutes of rest using an automated device (TM-2665P; Analog\&Digital Co., Ltd, Tokyo, Japan).

Blood samples were taken from the antecubital vein early in the morning after a $12 \mathrm{~h}$ overnight fast. Fasting plasma glucose, total cholesterol, high-density lipoprotein (HDL)-cholesterol, triglyceride and albumin were measured using a Hitachi 7600-110 chemistry autoanalyzer (Hitachi, Tokyo, Japan). C-reactive protein was measured using a latex-enhanced immunoturbidimetric method.

Total testosterone and sex hormone-binding globulin (SHBG) concentrations were measured using an electrochemiluminescence assay with a Modular Analytics E170 system (Roche Diagnostic Systems, Basel, Switzerland). The mean interassay coefficient of variation was $3.8 \%$ for total testosterone and $1.9 \%$ for SHBG. Bioavailable testosterone (BT) and free testosterone (FT) were calculated using the computerized Vermeulen formula, ${ }^{10}$ which is available on the International Society For The Study of the Aging Male web site (http://www. issam.ch).

\section{CCS measurement}

MDCT was performed to measure CCS (Philips Brilliance 64; Philips Medical System, Best, The Netherlands). A 40-80 mg propranolol hydrochloride was administered orally $1 \mathrm{~h}$ before the examination to reduce the heart rate in patients who had a heart rate more than 65 beats per min. We used a prospective ECG-gating protocol with a step-and-shoot technique. With the participants in the supine position, MDCT scanning was performed in the craniocaudal direction within a single breath-hold at end-inspiratory suspension. A $1.0 \mathrm{ml} \mathrm{kg}-1$ of iodinated contrast medium was administered intravenously. Imaging was performed by using a real time bolus tracking technique. The scans started $7 \mathrm{~s}$ after a trigger threshold of 110 Hounsfield units was reached. The breath-hold was achieved by all participants.

The image reconstruction was performed on the scanner's workstation using commercially available software (Extended Brilliance Workstation; Philips Medical System). Reconstructed images were analyzed for the presence and extent of coronary artery calcification by using coronary calcium quantification software (Aquarius Workstation; TeraRecon, Inc., Foster City, CA, USA). Total calcium score of all patients was calculated with dedicated software and expressed as Agatston scores. Agatston score is a commonly used scoring method that calculates the total amount of calcium on the basis of the number, areas, and peak Hounsfield units of the detected calcified lesions. $^{11}$

\section{Statistical analysis}

Demographic and biochemical characteristics of the study population were described as percentages for categorical variables, and as the mean \pm s.d. in the case of normal distribution and as median [25th percentile to 75 th percentile] or geometric mean \pm s.d. in the case of non-normal distribution. In addition, we divided the subjects into two groups as follows: the control $(\mathrm{CCS}=0)$ and the coronary calcification group $(\mathrm{CCS} \geqslant 1)$. The basic characteristics of each group were compared using the Student's $t$-test for continuous variables and the

Table 1 Characteristics of the study population

\begin{tabular}{|c|c|c|c|c|}
\hline & Total & Controls & Coronary calcification & $\mathrm{P}$ value \\
\hline Number & 291 & 186 & 105 & \\
\hline Age (year) & $52.8 \pm 9.3$ & $49.5 \pm 7.6$ & $58.7 \pm 9.3$ & $<0.001$ \\
\hline Body mass index $\left(\mathrm{kg} \mathrm{m}^{-2}\right)$ & $22.9 \pm 1.6$ & $23.0 \pm 1.5$ & $22.8 \pm 1.8$ & 0.394 \\
\hline Current smoker (\%) & 34.4 & 39.8 & 24.8 & 0.009 \\
\hline Alcohol consumption ${ }^{\mathrm{a}}(\%)$ & 25.4 & 30.7 & 16.2 & 0.006 \\
\hline Regular exercise $^{\mathrm{b}}(\%)$ & 58.4 & 58.1 & 59.1 & 0.870 \\
\hline Systolic blood pressure (mmHg) & $123.2 \pm 14.2$ & $121.9 \pm 13.7$ & $125.4 \pm 15.0$ & 0.046 \\
\hline Diastolic blood pressure (mmHg) & $77.8 \pm 8.7$ & $77.0 \pm 8.4$ & $79.1 \pm 9.2$ & 0.047 \\
\hline Fasting plasma glucose $\left(\mathrm{mg} \mathrm{dl}^{-1}\right)$ & $95.5 \pm 19.9$ & $93.1 \pm 18.3$ & $99.7 \pm 22.0$ & 0.009 \\
\hline Total cholesterol (mg dl${ }^{-1}$ ) & $191.4 \pm 35.1$ & $191.4 \pm 35.3$ & $191.5 \pm 34.8$ & 0.975 \\
\hline HDL-cholesterol (mg dl ${ }^{-1}$ ) & $49.7 \pm 10.8$ & $50.2 \pm 11.1$ & $48.8 \pm 10.1$ & 0.286 \\
\hline Triglyceride $\left(\mathrm{mg} \mathrm{dl}^{-1}\right)$ & $122.5 \pm 62.0$ & $119.7 \pm 59.2$ & $127.4 \pm 66.7$ & 0.307 \\
\hline C-reactive protein $\left(\mathrm{mg} \mathrm{I}^{-1}\right.$ ) & $2.2 \pm 5.7$ & $2.5 \pm 6.7$ & $1.7 \pm 3.0$ & 0.145 \\
\hline Albumin $\left(\mathrm{g} \mathrm{dl}^{-1}\right)$ & $4.7 \pm 0.3$ & $4.7 \pm 0.3$ & $4.7 \pm 0.3$ & 0.329 \\
\hline \multicolumn{5}{|l|}{ Male sex hormones (nmol I-1) } \\
\hline Total testosterone & $17.0[13.7-20.5]$ & $17.1[13.6-20.6]$ & 16.9 [13.9-20.3] & \\
\hline Sex hormone-binding globulin (SHBG) & $40.7[31.9-51.2]$ & $38.3[29.7-48.0]$ & $43.7[34.0-53.9]$ & \\
\hline Bioavailable testosterone (BT) & $7.29[5.97-9.26]$ & $7.42[6.32-9.85]$ & $6.89[5.72-8.40]$ & \\
\hline Free testosterone (FT) & $0.29[0.24-0.36]$ & $0.29[0.25-0.38]$ & $0.28[0.23-0.33]$ & \\
\hline
\end{tabular}

Abbreviation: HDL-cholesterol, high-density lipoprotein-cholesterol.

Data are expressed as the mean \pm s.d., proportions or median [25th percentile to 75 th percentile] for skewed variables. $P$ values were calculated with $t$-test or Chi-square test.

${ }^{a}$ Alcohol drinking $\geqslant 140$ g per week.

${ }^{\mathrm{b}}$ Regular exercise $\geqslant$ twice per week. 
Table 2 Univariate analysis of factors associated with coronary artery calcification

\begin{tabular}{|c|c|c|}
\hline Variables & Odds ratio $(95 \% \mathrm{Cl})$ & $\mathrm{P}$ value \\
\hline Age (year) & $1.14(1.10-1.18)$ & $<0.001$ \\
\hline Body mass index $\left(\mathrm{kg} \mathrm{m}^{-2}\right)$ & $0.93(0.81-1.08)$ & 0.364 \\
\hline Systolic blood pressure $(\mathrm{mmHg})$ & $1.02(1.00-1.04)$ & 0.048 \\
\hline Diastolic blood pressure $(\mathrm{mmHg})$ & $1.03(1.00-1.06)$ & 0.048 \\
\hline Fasting plasma glucose $\left(\mathrm{mg} \mathrm{dl}^{-1}\right)$ & $1.02(1.00-1.03)$ & 0.011 \\
\hline Total cholesterol $\left(\mathrm{mg} \mathrm{dl}^{-1}\right)$ & $1.00(0.99-1.01)$ & 0.975 \\
\hline HDL-cholesterol (mg dl ${ }^{-1}$ ) & $0.99(0.97-1.01)$ & 0.286 \\
\hline Triglyceride (mg dl ${ }^{-1}$ ) & $1.00(0.99-1.01)$ & 0.307 \\
\hline C-reactive protein $\left(\mathrm{mg} \mathrm{I}^{-1}\right)$ & $0.97(0.91-1.03)$ & 0.250 \\
\hline Total testosterone ${ }^{\mathrm{a}}\left(\mathrm{nmol} \mathrm{I}^{-1}\right)$ & $0.93(0.46-1.89)$ & 0.845 \\
\hline Sex hormone-binding globulin ${ }^{\mathrm{a}}(\mathrm{SHBG})\left(\mathrm{nmol} \mathrm{I}^{-1}\right)$ & $2.47(1.33-4.60)$ & 0.004 \\
\hline Bioavailable testosterone $^{\mathrm{a}}(\mathrm{BT})\left(\mathrm{nmol} \mathrm{I}^{-1}\right)$ & $0.44(0.21-0.92)$ & 0.030 \\
\hline Free testosterone $^{a}(\mathrm{FT})\left(\mathrm{nmol} \mathrm{I}^{-1}\right)$ & $0.42(0.20-0.89)$ & 0.024 \\
\hline
\end{tabular}

Abbreviation: HDL-cholesterol, high-density lipoprotein-cholesterol.

a Values have been analyzed after log-transformation. $P$ values were calculated with univariate logistic regression analysis.

chi-square test for categorical variables. Univariate regression analysis was performed to measure the strength of the relationship between coronary calcification and surrogate markers for CVD. Among subjects with coronary calcification, associations between sex hormone levels and CCS were evaluated using multiple linear regression analysis with CCS being a continuous variable after log-transformation of sex hormones and CCS. All analyses were conducted using SAS statistical software, version 9.1 (SAS Institute Inc, Cary, NC, USA). All statistical tests were two-sided, and statistical significance was determined with a $P$ value $<0.05$.

\section{RESULTS}

The mean subject age was $52.8 \pm 9.3$ years. The median serum concentration of total testosterone was $17.0 \mathrm{nmol}^{-1}$, SHBG was $40.7 \mathrm{nmol}$ $1^{-1}$, BT was $7.29 \mathrm{nmol}^{-1}$ and FT was $0.29 \mathrm{nmol}^{-1}$. The geometric mean CCS was $36.6 \pm 5.0$, which was calculated using the following equation: exp [mean (log CCS)]. All male sex hormones and CCS were positively skewed. Age, systolic blood pressure, diastolic blood pressure and fasting plasma glucose were higher in the group with coronary calcification $(P<0.001, P=0.046, P=0.047$ and $P=0.009$, respectively). The mean BMI was similar in both groups. The percentage of current smokers and alcohol consumption was higher in the control group ( $P=0.009$ and $P=0.006$, respectively) (Table 1).

According to univariate analysis, old age, high blood pressure, high fasting plasma glucose, high SHBG, low BT and low FT were associated with coronary artery calcification, whereas total testosterone was not
(Table 2). Upon multivariate linear regression analysis, BT and FT were found to be independently and inversely related with CCS after adjusting for age and BMI, whereas total testosterone and SHBG were not. We also assessed associations between male sex hormones and CCS after additional adjustment for smoking status, alcohol consumption, regular exercise, mean blood pressure, resting heart rate, C-reactive protein, fasting plasma glucose, total cholesterol, triglyceride, high-density lipoprotein-cholesterol, hypertension medication and hyperlipidemia medication. These inverse and independent associations remained for BT after using model 3 (Table 3 ).

\section{DISCUSSION}

In this cross-sectional study, BT was inversely associated with the degree of subclinical coronary artery calcification in non-obese men without clinical CVD but with some degree of coronary calcification. A decreased testosterone level has been found to be predictive of subclinical atherosclerosis in some observational studies. Most studies were performed to determine the relationship between testosterone and carotid artery intima-media thickness ${ }^{12-14}$ or abdominal aortic calcification. ${ }^{15}$ To our knowledge, there was only one previous study ${ }^{16}$ that focused on the association between sex hormone and subclinical coronary calcification. Ouyang et al. ${ }^{16}$ reported that testosterone is associated with coronary calcium score, but the study was conducted only on postmenopausal women.

Although the reason for the relationship between BT and CCS is unclear, it may be explained by some biological mechanisms. Endothelial dysfunction has been linked to testosterone deficiency. There are two possible mechanisms for this relationship: direct and indirect mechanisms. For the former, testosterone has positive effects on endothelial function by directly stimulating endothelium-derived nitric oxide. ${ }^{17,18}$ Additionally, testosterone could stimulate endothelial progenitor cells, which plays a key role in endothelial repair. ${ }^{19,20} \mathrm{Lu}$ et $a .^{21}$ have proposed that decreased testosterone is associated with ultrastructural damage of the aortic endothelium. In this regard, hypogonadism has been reported to contribute to the development of metabolic syndrome. ${ }^{22-24}$ Metabolic syndrome represents a cluster of cardiometabolic risk factors, including central obesity, elevated blood pressure, impaired glucose metabolism and atherogenic dyslipidemia. Thus, a low testosterone level could have an indirect deleterious effect on endothelium through induction of the metabolic syndrome. The relatively low cardiometabolic risk of the subjects in the present study suggests that the level of BT may affect the coronary arteries independently of the metabolic syndrome.

Our results demonstrate that FT was associated with the severity of CCS independently of age and BMI $(P=0.042)$, but not of other traditional CVD risk factors including blood pressure and fasting plasma

Table 3 Multiple regression analysis showing the independent contribution of sex hormones to coronary artery calcification in non-obese men with some degree of coronary calcification defined as coronary calcium score $\geqslant 1$

\begin{tabular}{|c|c|c|c|c|c|c|}
\hline & \multicolumn{2}{|c|}{ Model 1} & \multicolumn{2}{|c|}{ Model 2} & \multicolumn{2}{|c|}{ Model 3} \\
\hline & $\beta \pm s . e .^{b}$ & $\mathrm{P}$ value & $\beta \pm s . e .^{b}$ & $\mathrm{P}$ value & $\beta \pm s . e .^{b}$ & $\mathrm{P}$ value \\
\hline Total testosterone ${ }^{\mathrm{a}}\left(\mathrm{nmol} \mathrm{I} \mathrm{I}^{-1}\right)$ & $-0.713 \pm 0.535$ & 0.186 & $-0.596 \pm 0.517$ & 0.252 & $-0.225 \pm 0.535$ & 0.674 \\
\hline Sex hormone-binding globulin ${ }^{\mathrm{a}}(\mathrm{SHBG})\left(\mathrm{nmol} \mathrm{I}^{-1}\right)$ & $0.945 \pm 0.496$ & 0.059 & $0.365 \pm 0.540$ & 0.501 & $0.908 \pm 0.579$ & 0.121 \\
\hline Bioavailable testosterone $^{a}(\mathrm{BT})\left(\mathrm{nmol} \mathrm{I}^{-1}\right)$ & $-1.830 \pm 0.498$ & $<0.001$ & $-1.330 \pm 0.548$ & 0.017 & $-1.157 \pm 0.572$ & 0.046 \\
\hline Free testosterone $\mathrm{a}^{\mathrm{a}}(\mathrm{FT})\left(\mathrm{nmol} \mathrm{I}^{-1}\right)$ & $-1.674 \pm 0.532$ & 0.002 & $-1.156 \pm 0.563$ & 0.042 & $-0.983 \pm 0.595$ & 0.102 \\
\hline
\end{tabular}

a Values have been analyzed after log-transformation.

${ }^{b}$ Units of beta are log-unit increased coronary calcium score per 1 log unit greater sex hormone level. Model 1: unadjusted. Model 2: adjusted for age and body mass index. Model 3: adjusted for age, body mass index, smoking status, alcohol intake, exercise, mean blood pressure, resting heart rate, C-reactive protein, fasting plasma glucose, total cholesterol, triglyceride, HDL-cholesterol, hypertension medication and hyperlipidemia medication. 
glucose $(P=0.102)$. Although all blood samples were drawn early in the morning to minimize the diurnal variation in testosterone levels, ${ }^{25}$ FT might be less reliable rather than BT to represent basal androgenic activity.

However, our study has some limitations. First, our study is a cross-sectional study, and the cause-effect relationship between male sex hormones and CCS remains unclear. Second, we used calculated rather than directly measured BT and FT. Third, our results cannot be generalized to women. Fourth, the study population may not be representative of the general population of Korea because the study subjects were volunteers who visited a single hospital for physical check-up. Therefore, this study may have been affected by the selection bias. In this regard, the prevalence of current smoker was lower in subjects with coronary calcification. Fifth, only one sex hormone measurement was included in the analysis because the current study was based on the retrospective review. Therefore, we did not provide the intra-assay coefficient of variation. Lastly, bioavailable testosterone was inversely related to coronary calcification, but only in those with some degree of coronary calcification. Hence, the relevance of bioavailable testosterone to initiation of coronary calcification has not been addressed in this study.

In conclusion, our findings indicate that BT is inversely associated with subclinical coronary artery calcification in non-obese men without clinical CVD but with some degree of coronary calcification. Accordingly, early detection of decreased BT is important in the assessment of potential cardiovascular risk and could be an initiative for CVD prevention and vascular health management.

\section{AUTHOR CONTRIBUTIONS}

BJP performed the statistical analysis and drafted the manuscript. JYS participated in its design and coordination. YJL participated in the design of the study and performed the statistical analysis. JHL participated in data collection and management. HRL conceived of the study, and participated in its design and coordination and helped to draft the manuscript. All authors read and approved the final manuscript.

\section{COMPETING FINANCIAL INTERESTS}

The authors declare that they have no competing financial interests.

1 Smith SC Jr, Jackson R, Pearson TA, FusterV Yusuf S et al. Principles for national and regional guidelines on cardiovascular disease prevention: a scientific statement from the World Heart and Stroke Forum. Circulation 2004; 109: 3112-21.

2 Budoff MJ, Gul KM. Expert review on coronary calcium. Vasc Health Risk Manag 2008; 4: 315-24.
3 Ma ES, Yang ZG, Li Y, Dong ZH, Zhang L et al. Correlation of calcium measurement with low dose 64-slice CT and angiographic stenosis in patients with suspected coronary artery disease. Int J Cardiol 2008; 140: 249-52.

4 Greenland P, LaBree L, Azen SP, Doherty TM, Detrano RC. Coronary artery calcium score combined with Framingham score for risk prediction in asymptomatic individuals. JAMA 2004; 291: 210-5.

5 Simon D, Charles MA, Nahoul K, Orssaud G, Kremski J et al. Association between plasma total testosterone and cardiovascular risk factors in healthy adult men: The Telecom Study. J Clin Endocrinol Metab 1997: 82: 682-5.

6 Khaw K, Dowsett M, Folkerd E, Bingham S, Wareham N et al. Endogenous testosterone and mortality due to all causes, cardiovascular disease, and cancer in men: European prospective investigation into cancer in Norfolk (EPIC-Norfolk) prospective population study. Circulation 2007; 116: 2694-701.

7 Malkin CJ, Pugh PJ, Morris PD, Asif S, Jones TH et al. Low serum testosterone and increased mortality in men with coronary heart disease. Heart 2010; 96: 1821-5.

8 Phillips GB, Pinkernell BH, Jing TY. The association of hypotestosteronemia with coronary artery disease in men. Arterioscler Thromb 1994; 14: 701-6.

9 World Health Organization, International Association for the Study of Obesity, International Obesity Task Force. The Asia-Pacific perspective: redefining obesity and its treatment. Sydney NSW: Health Communications Australia; 2000.

10 Vermeulen A, Verdonck L, Kaufman JM. A critical evaluation of simple methods for the estimation of free testosterone in serum. J Clin Endocrinol Metab 1999; 84: 3666 72 .

11 Agatston AS, Janowitz WR, Hildner FJ, Zusmer NR, Viamonte M Jr et al. Quantification of coronary artery calcium using ultrafast computed tomography. J Am Coll Cardiol 1990; 15: 827-32.

12 Mäkinen J, Jarvisalo M, Pollanen P, Perheentupa A, Irjala K et al. Increased carotid atherosclerosis in andropausal middle-aged men. J Am Coll Cardiol 2005; 45: 1603

13 Fukui M, Kitagawa Y, Nakamura N, Kadono M, Mogami S et al. Association between serum testosterone concentration and carotid atherosclerosis in men with type 2 diabetes. Diabetes Care 2003; 26: 1869-73.

14 Muller M, van den Beld AW, Bots ML, Grobbee DE, Lamberts SW et al. Endogenous sex hormones and progression of carotid atherosclerosis in elderly men. Circulation 2004; 109: 2074-9.

15 Hak A, Witteman J, de Jong F, Geerlings M, Hofman A et al. Low levels of endogenous androgens increase the risk of atherosclerosis in elderly men: the Rotterdam study. $J$ Clin Endocrinol Metab 2002; 87: 3632-9.

16 Ouyang P, Vaidya D, Dobs A, Golden SH, Szklo M et al. Sex hormone levels and subclinical atherosclerosis in postmenopausal women: the Multi-Ethnic Study of Atherosclerosis. Atherosclerosis 2009; 204: 255-61.

17 Yue P, Chatterjee K, Beale C, Poole-Wilson PA, Collins P. Testosterone relaxes rabbit coronary arteries and aorta. Circulation 1995; 91: 1154-60.

18 Miller VM, Mulvagh SL. Sex steroids and endothelial function: translating basic science to clinical practice. Trends Pharmacol Sci 2007; 28: 263-70.

19 Foresta C, Caretta N, Lana A, DeToni L, Biagioli A et al. Reduced number of circulating endothelial progenitor cells in hypogonadal men. J Clin Endocrinol Metab 2006; 91: 4599-602.

20 Foresta C, Zuccarello D, de Toni L, Garolla A, Caretta N et al. Androgens stimulate endothelial progenitor cells through an androgen-mediated pathway. Clin Endocrinol 2008: 68: 284-9.

21 Lu YL, Kuang L, Zhu H, Wu H, Wang XF et al. Changes in aortic endothelium ultrastructure in male rats following castration, replacement with testosterone and administration of 5-alpha-reductase inhibitor. Asian J Androl 2007: 9: 843-7.

22 Mohr BA, Bhasin S, Link CL, O'Donnell AB, McKinlay JB. The effect of changes in adiposity on testosterone levels in older men: longitudinal results from the Massachusetts Male Aging Study. Eur J Endocrinol 2006; 155: 443-52.

23 Kalyani RR, Dobs AS. Androgen deficiency, diabetes, and the metabolic syndrome in men. Curr Opin Endocrinol Diabetes Obes 2007; 14: 226-34.

24 Rodriguez A, Muller DC, Metter EJ, Maggio M, Harman SM et al. Aging, androgens, and the metabolic syndrome in a longitudinal study of aging. J Clin Endocrinol Metab 2007; 2; 3568-72.

25 Brambilla DJ, Matsumoto AM, Araujo AB, McKinlay JB. The effect of diurnal variation on clinical measurement of serum testosterone and other sex hormone levels in men. $J$ Clin Endocrinol Metab 2009; 94: 907-13. 